DOI: 10.12731/2227-930X-2020-2-38-47

УДК 004.94

\title{
ОБ ОПТИМИЗАЦИИ СИСТЕМЫ ПЕРЕВОЗОК НА ОСНОВЕ МОНИТОРИНГОВОЙ ИНФОРМАЦИИ
}

Львович Я.Е., Преображенский А.П., Чопоров О.Н.

В статье рассматривается задача, в которой требуется оптимизировать систему перевозок. При этом необходимо опираться на мониторинговую информацию. Приведена схема работы сетевых компонентов системы перевозок при учете мониторинговой информации.

Ключевые слова: система перевозок; мониторинговая информацчия; оптимизацчия.

\section{ABOUT OPTIMIZATION OF THE TRANSPORTATION SYSTEM BASED ON MONITORING INFORMATION}

\section{Lvovich Ya.E., Preobrazhenskiy A.P., Choporov O.N.}

The paper considers the problem in which it is required to optimize the transportation system. It is necessary to rely on monitoring information. The scheme of the network components of the transportation system with the monitoring information is given.

Ключевые слова: transportation system; monitoring information, optimization.

\section{Введение}

Решение проблемы повышения эффективности управления функционированием организаций, связанных с перевозками, может осуществляться на базе объединения при помощи управляющего центра в сетевые структуры. При этом необходимо обращать внимание на возможности моделирования и оптимизации, 
чтобы были обеспечены требования, предъявляемые к показателям качества работы организаций $[1,2]$. Для существующих условий цифровизации происходит формирование предпосылок по их эффективному применению, когда существует информационный мониторинг. Используются разные способы, связанные с оцениванием того насколько будут соответствовать режимы качественной работе компонентов, входящих в состав сетевых структур организации перевозок требуемым нормам [3, 4]. Информационный мониторинг оказывает влияние на то, какие будут применяться подходы в математическом моделировании. Сама структура информационного мониторинга определяется особенностями того, каким образом происходит упорядоченность компонентов цифровой трансформации оценок качественной работы сетевых компонентов организации перевозок [5]. Она имеет двухуровневый характер. С другой стороны, взаимодействие администрации сетевых объектов с администрацией управляющего центра и потребителем при определении ресурсов качественного функционирования требует ориентации разрабатываемых методов принятия управленческих решений на различные формы экспертного оценивания [6]. Особенности комбинации указанных характеристик могут быть проявлены на любом из уровней моделирования. За счет того, что формируются процедуры оптимизации, будет наблюдаться статистический характер в среде информационного мониторинга. Это происходит вследствие того, что внутрь рандомизированной поисковой среды будет происходить ввод переменных, относящихся к оптимизационным задачам $[7,8]$. Для итогового шага в ходе оптимизации необходимо осуществлять выделение объектов, связанных с ресурсным обеспечением. Тогда будет обеспечиваться соответствующий вариант по управлению работой объектов в рамках требуемых норм.

Особенности компонентов организации, связанной с перевозки с точки зрения наблюдения их внутри среды информационного мониторинга. Сетевыми объектами системы, связанной с перевозками, мы будем считать автономные структуры, 
имеющие однородный характер работы. Они группируются в организационное целое. При этом происходит исполнение тех целей, которые были заданы. В качестве одной из обозначаемых целей, чтобы происходил процесс объединения, можно указать то, что есть требование по качественной работе компонентов перевозок. Есть нумерационное множество $i=\overline{1, I}$, характеризующее их совокупность.

Информационный мониторинг связан с активной цифровизацией систем перевозок. В мониторинговом оценивании можно указать виды:

- проведение административного оценивания по соответствию режимов качественной работы компонентов и соответствующих результатов нормам;

- проведение количественного оценивания по факторам $x_{i j}$ Они описывают режимы качественной работы компонентов, здесь $l=\overline{1, L}-$ нумерационное множество направлений работы; $j_{l}=\overline{1, J_{l}}-$ нумерационное множество, показывающее факторы;

- проведение процессов количественного оценивания удовлетворенности клиентов результатами работы компонентов $y_{i n 1}$ здесь $n_{1}=\overline{1, N_{1}}-$ является нумерационным множеством показателей. Они демонстрируют то, какая потребительская оценка в качественной работе;

- проведение вопросно-ответного оценивания удовлетворенности клиентов качеством процесса функционирования объектов $y_{i n_{2}} m g$, где $m=\overline{1, M}$ - нумерационное множество направлений оценивания; $n_{2 m}=\overline{1, N_{2 m}}-$ нумерационное множество вопросов по $m$-му направлению; $g_{n_{2} m}=\overline{1, G_{n_{2} m}}-$ нумерационное множество ответов.

При цифровой трансформации данных соответствующих типов видов мониторингового оценивания можно отметить:

1. Охват всех аспектов, характеризующих режимы работы сетевых компонентов, представляющих собой множество распределенных объектов системы перевозок.

2. Создаются предпосылки, чтобы ставить и решать задачи, связанные с оптимизацией процессов управления на основе выбора 
факторов $x_{i j l}$ и их значений, распределением обеспечения ресурсов, которые дают возможности для выполнения требований относительно критериев клиентов $y_{i n 1}, y_{i n 2}$.

Какие они бывают? Какие они бывают? Отметим следующие: результативно-нормативные - результаты соответствуют нормативным требованиям; процессуально-нормативные - факторы, связанные с режимами работы, нормативным требованиям; результативно-потребительские - результаты соответствуют требованиям клиентов; процессуально-потребительские - процессы работы компонентов системы потребностям клиентов. Таким образом, совокупность цифровых ресурсов образует многоаспектную среду информационного мониторинга. Схема работы сетевых компонентов системы перевозок при учете того, что осуществляется информационный мониторинг, дана на рис. 1. Видно, что идет взаимодействие сетевых объектов и управляющего центра в системе управления перевозками и клиентов $r_{i}, i=\overline{1, I}$. В центре осуществляется оценка по полученным показателям качества работы и происходит определение относительно ресурсного обеспечения $V_{i}, i=\overline{1, I}$, чтобы были сформированы условия по выполнению требований относительно показателей.

Управленческое решение будет принято такое, в которое будут входить факторы, являющиеся значимыми. При этом необходимо вести управление по распределенному ресурсному обеспечению $V_{i}$.

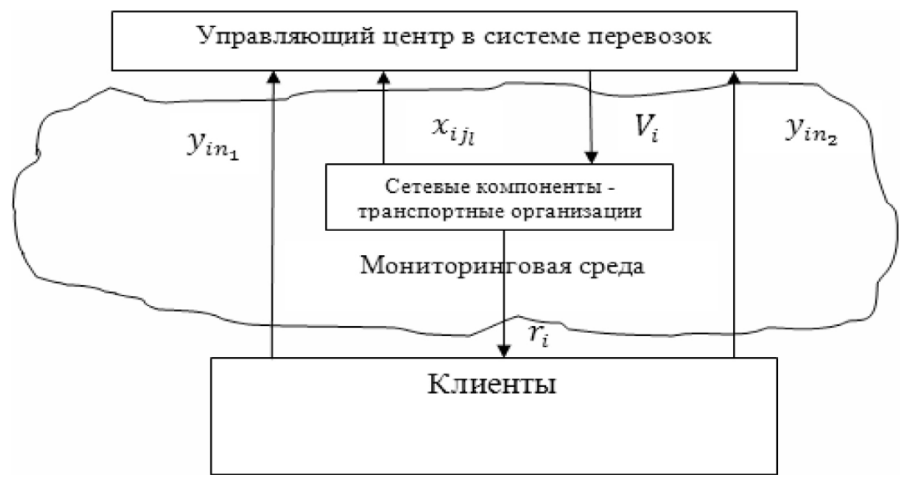

Рис. 1. Схема работы сетевых компонентов системы перевозок при учете мониторинговой информационной среды 
Особенности оптимизации ресурсов при управлении работой компонентов системы перевозок. Процессы оптимизации ресурсов в ходе управления качеством работы компонентов системы перевозок ведется относительно двух направлений:

- проведение оптимального распределения по целевому ресурсу $V_{i}$ (рис. 1), который выделяется от управляющего центра, чтобы достигать приоритетные условия качественной работы в $i$-м сетевом компоненте;

- процессы привлечения дополнительных ресурсов.

Для решения задач необходимо привлекать теорию моделирования и оптимизации в организационных системах.

При распределении $j=\overline{1, J}$ среди условий $U_{j}, j=\overline{1, J}$ качественной работы в $i$-м компоненте $V_{i}$ исходят из определенных локальных функций $Y_{j}\left(v_{i j}\right)$. Решение задачи оптимизации связано с определением распределения ресурсов $V$, дающих максимизацию общего эффекта. Отметим два случая, когда ведется оценка информации в мониторинге (рис. 1):

- в данных $x_{i j l}, y_{i n 1}, y_{i n 2}$ достоверным образом оценивается работа компонентов;

- данные $x_{i j l}, y_{i n 1}, y_{i n 2}$ рассматриваются как недостаточно достоверные.

Для первого случая оптимизационную задачу записываем так:

$$
\begin{gathered}
\sum_{j=1}^{J} Y_{j}\left(v_{i j}\right) \rightarrow \max \\
\sum_{j=1}^{J} v_{i j} \leq V_{i}, \quad v_{i j} \geq 0, j=\overline{1, J} .
\end{gathered}
$$

Задача (1) решается при функции $Y_{j}\left(v_{i j}\right)=2 \sqrt{\alpha_{j} v_{i j}}$, здесь $a_{j}-$ показатель, демонстрирующий эффективность применения ресурсов при поддержке $j$-го условия, когда достигается норма работы. Для оптимального решения получаем

$$
v_{i j}=\frac{\alpha_{j}}{H} V_{i}, j=\overline{1, J}
$$




$$
H=\sum_{J=1}^{J} \alpha_{j} .
$$

Для второго случая идет формирование собственной оценки $\alpha_{j}^{\prime}$ . Если $\alpha_{j}^{\prime} \neq \alpha_{j}$ тогда решение (1) может получиться достаточно отличающимся от (2). Тогда для получения достоверной информации по работе компонента вводится плата относительно единицы предоставляемого ресурса. Тогда имеем такую целевую функцию по $j$-му компоненту

$$
Y_{j}\left(v_{i j}\right)-\rho v_{i j}=2 \sqrt{\alpha_{j} v_{i j}}-\rho v_{i j} .
$$

Максимум (3) будет, когда

Делая выбор $\rho$ по условию

$$
v_{i j}=\frac{\pi_{j}}{\rho^{2}}
$$

$$
\sum_{j=1}^{J} v_{j} \leq V_{i}
$$

имеем

$$
\rho=\sqrt{\frac{H}{V_{i}}} .
$$

Делая подстановку $a_{i}$ вместо оценки $\alpha_{j}^{\prime}$, а вместо $H$, будем иметь

$$
H^{\prime}=\sum_{j=1}^{J} \alpha_{i}^{\prime},
$$

получим распределение ресурса $V_{i}$, базируясь на оценках управления $\alpha_{j}^{\prime}$

$$
v_{j}=\frac{\alpha_{j}^{\prime}}{H^{\prime}} V, \rho=\sqrt{\frac{H^{\prime}}{V_{i}}} .
$$

Сравнивая результаты (2) и (5) мы можем сделать вывод о том, что следует повышать достоверность информации в мониторинге при оптимизации управления работы компонентов системы перевозок.

Поскольку ресурсы ограничены, тогда можно опираться на такие принципы распределения: принцип, связанный с пропорцио- 
нальным распределением, принцип, связанный с обратными приоритетами.

В первом из них в $j$-м условии получаем тот ресурс $v_{j}^{0}, j=\overline{1, J}$, который требуется когда сумма по всем условиям на получение объема ресурса будет соотноситься с ограничением (5). При не выполнении ограничения (5) идет распределение объемов среди условий пропорционально запрашиваемому количеству.

Во втором принципе основываются на следующем: будет тем выше приоритет в $j$-м условии, когда распределяется ресурс $V$, чем будет требоваться меньшее значение объема $v_{i}$. При этом происходит учет отдачи условия, когда растет эффективность работы. Она оценивается в ходе рассмотрения мониторинговой информации на основе величины потенциала $a_{i}$.

\section{Выводы}

Проведен анализ особенностей компонентов организации, связанной с перевозки с точки зрения наблюдения их внутри среды информационного мониторинга. Показаны характеристики оптимизации ресурсов при управлении работой компонентов системы перевозок.

Информация о конфликте интересов. Авторы заявляют об отсутствии конфликта интересов.

Информация о спонсорстве. Исследование не имело спонсорской поддержки.

\section{Сиисок литературы}

1. Лысанов Д.М., Бикмухаметова Л.Т. Анализ показателей качества и конкурентоспособности оборудования // International Journal of Advanced Studies. 2018. T. 8. № 4-2. C. 50-55.

2. Преображенский Ю.П. Построение складской структуры предприятия // Молодежь и системная модернизация страны Сборник научных статей 3-й Международной научной конференции студентов и молодых ученых. В 4-х томах. Ответственный редактор А.А. Горохов. 2018. С. 286-290. 
3. Преображенский Ю.П. О повышении эффективности работы промышленных предприятий // Исследование инновационного потенциала общества и формирование направлений его стратегического развития. Сборник научных статей 8-й Всероссийской научно-практической конференции с международным участием. 2018. C. 45-48.

4. Сапожникова С.М. Корпоративное управление в железнодорожном транспорте // International Journal of Advanced Studies. 2019. T. 9. № 4. С. 19-42.

5. Преображенский Ю.П. Проблемы управления в производственных организациях // Актуальные проблемы развития хозяйствующих субъектов, территорий и систем регионального и муниципального управления Материалы XIII международной научно-практической конференции. Под редакцией Ю.В. Вертаковой. 2018. С. 208-211.

6. Пеньков П.В. Экспертные методы улучшения систем управления // Вестник Воронежского института высоких технологий. 2012. № 9. С. 108-110.

7. Берман Н.Д., Белов А.М. Общественный транспорт и инновации // International Journal of Advanced Studies. 2019. T. 9. № 2. C. 7-13.

8. Шакиров А.А., Зарипова Р.С. Особенности моделирования логистических систем // International Journal of Advanced Studies. 2019. T. 9. № 4. C. 27-31.

\section{References}

1. Lysanov D.M., Bikmuhametova L.T. International Journal of Advanced Studies. 2018. V. 8. № 4-2, pp. 50-55.

2. Preobrazhenskij Yu.P. Molodezh' i sistemnaya modernizaciya strany Sbornik nauchnyh statej 3-j Mezhdunarodnoj nauchnoj konferencii studentov i molodyh uchenyh [Youth and systemic modernization of the country Collection of scientific articles of the 3rd International Scientific Conference of Students and Young Scientists]. Ed. A.A. Gorohov. 2018, pp. 286-290.

3. Preobrazhenskij Yu.P. Issledovanie innovacionnogo potenciala obshchestva i formirovanie napravlenij ego strategicheskogo razviti- 
ya. Sbornik nauchnyh statej 8-j Vserossijskoj nauchno-prakticheskoj konferencii s mezhdunarodnym uchastiem [Study of the innovative potential of society and the formation of directions for its strategic development. Collection of scientific articles of the 8th All-Russian Scientific and Practical Conference with international participation]. 2018, pp. 45-48.

4. Sapozhnikova S.M. International Journal of Advanced Studies. 2019. V. 9. № 4, pp. 19-42.

5. Preobrazhenskij Yu.P. Aktual'nye problemy razvitiya hozyajstvuyushchih sub"ektov, territorij i sistem regional 'nogo i municipal'nogo upravleniya Materialy HIII mezhdunarodnoj nauchno-prakticheskoj konferencii [Actual problems of the development of business entities, territories and systems of regional and municipal management Materials of the XIII international scientific and practical conference]. Ed. Yu.V. Vertakova. 2018, pp. 208-211.

6. Pen'kov P.V. Vestnik Voronezhskogo instituta vysokih tekhnologij. 2012. № 9, pp. 108-110.

7. Berman N.D., Belov A.M. International Journal of Advanced Studies. 2019. V. 9. № 2, pp. 7-13.

8. Shakirov A.A., Zaripova R.S. International Journal of Advanced Studies. 2019. V. 9. № 4, pp. 27-31.

\section{ДАННЫЕ ОБ АВТОРАХ}

Львович Яков Евсеевич, профессор, доктор технических наук, профессор

Федеральное государственное образовательное учреждение выстего образования Воронежский государственньй технический университет

ул. 20 лет Октября, 84, г. Воронеж, 394006, Российская Федерачия

Komkovvivt@yandex.ru

Преображенский Андрей Петрович, профессор, доктор технических наук, доцент 
Автономная некоммерческая образовательная организачия высшего образования Воронежский институт высоких технологий

ул. Ленина, 73a, г. Воронеж, 394043, Российская Федеращия Komkovvivt@yandex.ru

Чопоров Олег Николаевич, профессор, доктор технических наук, профессор Федеральное государственное образовательное учреждение выстего образования Воронежский государственный технический университет

ул. 20 лет Октября, 84, г. Воронеж, 394006, Российская Федерация

Komkovvivt@yandex.ru

\section{DATA ABOUT THE AUTHORS}

Lvovich Yakov Yevseevich, Professor, doctor of technical Sciences, Professor

Voronezh State Technical University

20 years of October Str., 84, Voronezh, 394006, Russian Federation Komkovvivt@yandex.ru

ORCID:0000-0002-7051-3763

Preobrazhenskiy Andrey Petrovich, professor, doctor of technical sciencies, associate professor Voronezh Institute of High Technologies

73a, Lenin Str., Voronezh, 394043, Russian Federation Komkovvivt@yandex.ru ORCID: 0000-0002-6911-8053

Choporov Oleg Nikolaevich, Professor, doctor of technical Sciences, Professor

Voronezh State Technical University

20 years of October Str., 84, Voronezh, 394006, Russian Federation Komkovvivt@yandex.ru

ORCID: 0000-0002-3176-499X 